Ophthalmologe 2022 $1119: 1064-1066$ https://doi.org/10.1007/s00347-021-01566-3 Eingegangen: 2. Dezember 2021 Überarbeitet: 10. Dezember 2021 Angenommen: 20. Dezember 2021 Online publiziert: 13. Januar 2022 ๑ Der/die Autor(en) 2022

\section{Okuläres Trauma durch eine Wühlmausschussfalle}

\author{
Lisa Lüdtke (D) · Rico Großjohann · Allam Tayar · Andreas Stahl · Frank Tost \\ Klinik für Augenheilkunde, Universitätsmedizin Greifswald, Greifswald, Deutschland
}

\begin{abstract}
Anamnese
Über den Notarzt wurde ein 71-jähriger Patient mit einer Schussverletzung durch eine Wühlmausschussfalle im Bereich der rechten Gesichtshälfte und des rechten Auges vorgestellt. Er berichtete über eine subjektive Sehverschlechterung und ein erhebliches Fremdkörpergefühl am betroffenen Auge. Im Bereich der rechten Gesichtshälfte habe es initial stärker geblutet. Die Blutung sei aber schnell zum Stillstand gekommen. Der Patient hatte bis auf eine mit Brille gut korrigierte Hyperopie keine bekannten ophthalmologischen Vorerkrankungen.
\end{abstract}

\section{Befunde}

Visus bei Aufnahme: auf dem betroffenem rechten Auge 0,16 ohne Korrektur und links 0,8 mit Korrektur von $+1,75 \mathrm{sph}$. (Sphäre) -0,75 cyl. (Zylinder) Achse $113^{\circ}$. Makroskopisch zeigten sich multiple gröbere Einsprengungen, Schmauchpartikel, Rötung und leichte Schwellung der rechten Gesichtshälfte. Besondere Beteiligung der periokulären Region, zusätzlich nur biomikroskopisch sichtbare flächenhafte Einbeziehung der gesamten Augenoberfläche im Lidspaltenbereich (- Abb. 1). Multiple tief eingesprengte Fremdkörper (FK) und Kontusionsfolgen mit Hyposphagma. Ausschluss intraokularer FK oder Gewebedefekte im Vorderabschnitt. Am Augenhintergrund kein Hinweis auf eine kontusionelle Netzhautschädigung bei dichter Synchisis nivea. Als bildgebendes Verfahren der Wahl wurden zur weiteren Detektion von etwaigen FK der multimodale Ultraschall (• Abb. 2) und die Vorderabschnitts-OCT(Okuläre Kohe- renztomografie) (• Abb. 3a und 4) ohne zusätzlichen FK-Nachweis eingesetzt.

\section{Diagnose}

Multiple Mikropartikel (FK-Einsprengungen) in Hornhaut und Bindehaut, leichte Contusio bulbi des rechten Auges durch Kartuschenmunition einer Wühlmausschussfalle.

\section{Therapie und Verlauf}

Intensive Spülung des Auges mit physiologischer Natriumchloridlösung sowie die Entfernung aller oberflächlichen FK im Bereich der Hornhaut und der Bindehaut. Antibiotische Lokaltherapie mit OfloxacinAugensalbe 2-stündlich am rechten Auge. Zur weiteren Einschätzung der FK erfolgte eine OCT des Vorderabschnitts. Es zeigten sich multiple bis zu $300 \mu \mathrm{m}$ tief eingedrungene große und kleine FK, die ohne größere Gewebetraumatisierung nicht zu entfernen waren (- Abb. 3a). Im weiteren Verlauf war das Stromaödem rückläufig, und die Hornhaut klarte auf. Bei reizfreiem Untersuchungsbefund (- Abb. 4) und Visusanstieg auf $0,8\left(+1,25-0,75 / 41^{\circ}\right)$ wurde auf die weitere Entfernung von FK verzichtet und die langfristige Befundkontrolle veranlasst. Vier Monate später war der Visus stabil. OCT-morphologisch war eine feine Narbenbildung zu beobachten, einige FK waren nicht mehr nachweisbar oder hatten an Größe verloren (- Abb. 5 und $3 b$ ).

\section{Diskussion}

In Deutschland gehören Schussverletzungen im Allgemeinen und im Fachbereich 


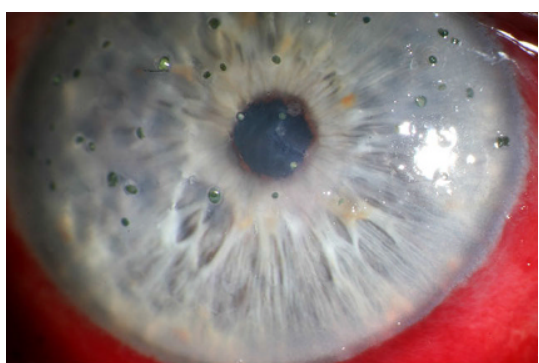

Abb. 1 \& Initialer Befund mit multiplen Fremdkörpereinsprengungen, Epithelschäden und Stromaödem der Hornhaut sowie Hyposphagma der Bindehaut am rechten Auge

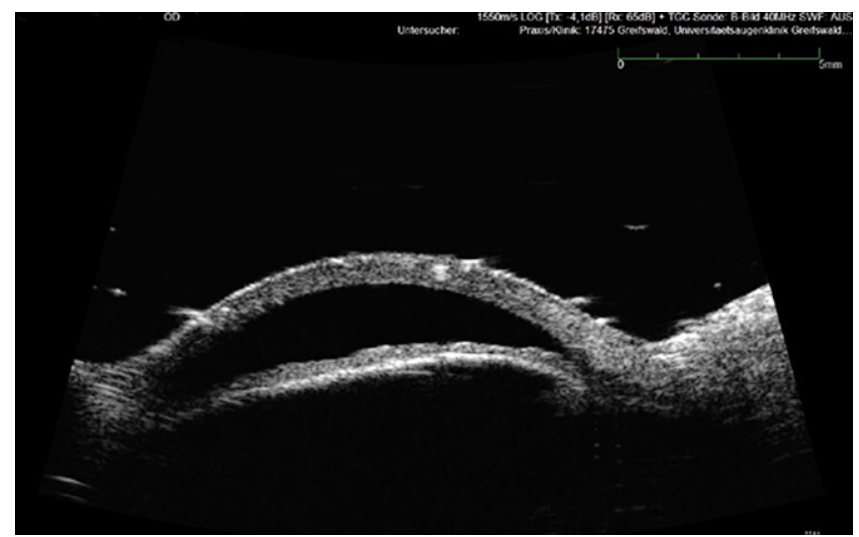

Abb. $2 \triangleleft$ Darstellung des Vorderabschnittes sowie der intrastromalen Fremdkörpereinsprengungen der Hornhaut mittels multimodalen UItraschalls
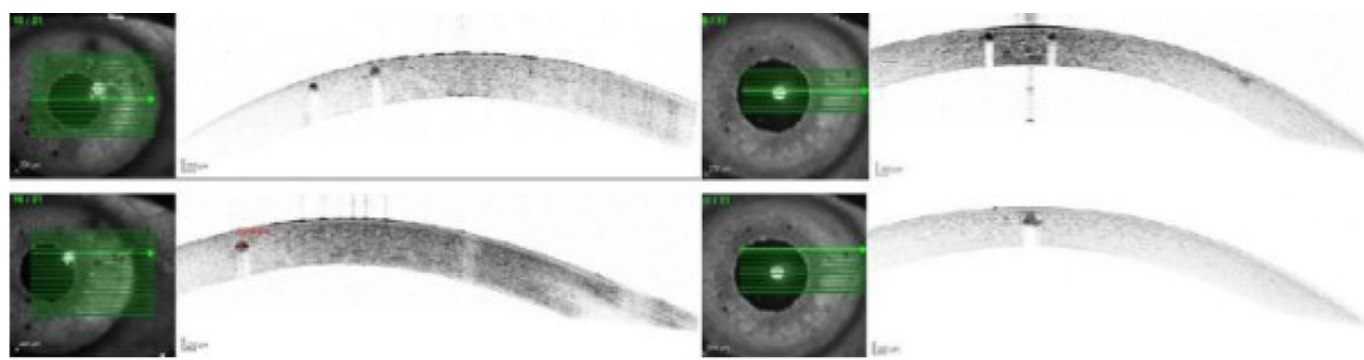

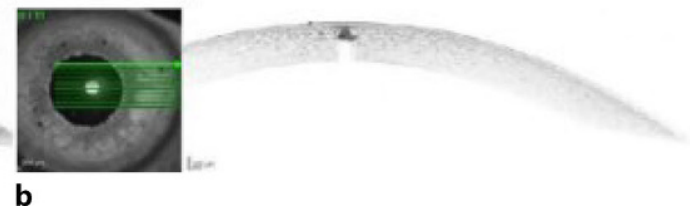

Abb. $3<$ a Die OCTDarstellung des Vorderabschnittes links zeigt die intrastromalen Fremdkörper sowie ihre Eindringtiefe in die Hornhaut. b OCTVorderabschnitt nach 4 Monaten mit im Vergleich zur Erstuntersuchung reduzierter Fremdkörpergröße der Ophthalmologie im Besonderen zu den seltenen klinischen Diagnosen im Unterschied zu anderen Ländern [12]. Dies beruht auf der starken Reglementierung von Schusswaffen und Munition durch das deutsche Waffengesetz. Die Wühlmausschussfalle zählt aufgrund der Verwendung von Platzpatronen (Kartuschenmunition) nicht zu den "scharfen“ Waffen. Der Zugang und Erwerb von Kartuschenmunition ist in Anlage 1 des Waffengesetzes geregelt. Nach Prüfung und Zulassung durch das Physikalisch-technische Bundesamt ist der Erwerb in Deutschland für jedermann ab 18 Jahren möglich [1]. Wühlmausschussfallen sind selbstauslösende Waffen zur Bekämpfung schädlicher Nagetiere, die offiziell geprüft und zugelassen sind. Technisch handelt es sich um stationäre Federpistolen, die mit Platzpatronen des Kalibers $9 \times 17$ betrieben werden. Dazu wird das Schussgerät über ein schraubbares Patronenlager geladen und in den Bau der Wühlmaus eingegraben. Der Schuss wird infolge leichter Berührung durch die Wühlmaus an einem Metallring vor der Mündung ausgelöst. Die Wühlmaus stirbt durch den dabei ausgelösten Hochdruckgasstrahl [4]. Handhabungsprobleme können zu Verletzungen beim Anwender füh- ren. Das Verletzungsmuster ist vergleichbar zu anderen Hochgeschwindigkeitsverletzungen mit multiplen FK wie der Schrotschussmunition. Die Schwere der Verletzung ist abhängig von der übertragenen Energie und somit von der Distanz, der Temperatur, der Pulvermenge, der Kugelgröße und -form, des Kugelgewichtes sowie vom Kugelquerschnitt. Daher muss bei diesen Verletzungen prinzipiell mit zusätzlichen schweren intraokularen Verletzungen gerechnet werden [11]. Bereits tief in die Kornea eingedrungene FK können durch Vernarbungen oder irreguläre Oberfläche dauerhafte Visuseinschränkungen verursachen [6, 7]. Sehr kleine Partikel hinterlassen häufig von außen nicht feststellbare Wunden, da sich das Gewebe über dem Defekt umgehend schließt. Eine zusätzliche Bildgebung ist unerlässlich [6] und sollte mittels Sonographie der Augen und Orbita erfolgen, ggf. stehen CT oder MRT zur Verfügung $[5,6,9]$. In der konkreten Behandlungssituation schloss sich eine Ultraschalluntersuchung an. In der sonographischen Diagnostik von FKimponieren Plastikpartikel als Objekte mit einer niedrigeren Echogenität als die Retina oder der Glaskörper und ohne Schallschatten. AndereStoffe, wiez. B. Metalle, Holz oder Glas, weisen eine höhere oder ähnliche Echogenität im Vergleich zum Umgebungsgewebe auf und rufen ggf. einen Schallschatten hervor [8]. Für eine gute optische Rehabilitation sollten so viele FK wie möglich entfernt werden. Die Entfernung muss bei Beteiligung der zentralen optischen Zone der Kornea gewebeschonend erfolgen. Je tiefer und größer eine Hornhautverletzung ist, desto größer ist das Risiko einer Narbenbildung [10]. Bei tief liegenden, aber inerten FK wie Glas oder Kunststoff muss nicht zwingend eine sofortige Entfernung erfolgen. Eine Verlaufskontrolle bezüglich etwaiger Störungen im Heilungsverlauf ist nötig $[6,7]$. Eine Vorderabschnitts-OCT unterstützt die Beurteilung der Lage der FK in der Tiefe bei der Indikationsstellung und Planung notwendiger intrakornealer FK-Entfernungen [3]. Bei großen, tiefen und zentralen Defekten empfiehlt sich eine häufigere Applikation der antibiotischen Lokaltherapie. Es sollte eine Reevaluation des Befundes und der Therapie insbesondere bei Verletzungen durch metallische und organische Partikel erfolgen $[2,6]$. Bei Funktionserholung und Beschwerdefreiheit verzichteten wir in Rücksprache mit dem Patienten auf eine vollständige FK-Entfernung. Erforderlich sind dann die 


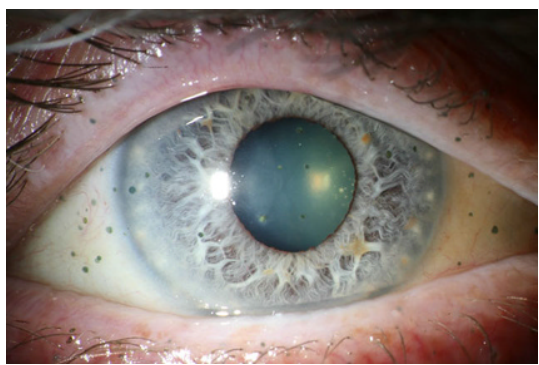

Abb. $4 \Delta$ Es zeigt sich ein reizfreier Befund 1,5 Wochen nach Trauma mit weiterhin bestehenden Fremdkörpereinsprengungen von Hornhaut und Bindehaut. Der Visus liegt bei 0,8

langfristige Befundkontrolle und Fortführung der topischen Steroidtherapie zur Reduktion der Narbenbildung [6]. Nach 4 Monaten fiel eine leicht rückläufige Anzahl von FK sowohl in der Hornhaut als auch in der Bindehaut auf. Demnach war das Material teilweise abbaubar, oder es kam zur Extrusion. Die Rückfrage beim Hersteller der Kartuschenmunition ergab kejnen konkreten Aufschluss. Das Treibmittel besteht aus einem Gel, welches auskristallisiert und verschiedene Komponenten enthält, u.a. Nitroglycerin. Die Verbrennungsprodukte sind laut Hersteller nicht näher untersucht worden, sodass sich die Zusammensetzung der Einsprengungen, deren Eigenschaften und Verhalten zum aktuellen Zeitpunkt nicht vollständig aufklären ließen.

\section{Fazit}

- Hochgeschwindigkeitsverletzungen des Auges mit multiplen FK erfordern eine multimodale Diagnostik und Therapie.

- Die intraokulare Beteiligung durch FK ist auszuschließen, Hinweise sind flache Vorderkammer, niedriger Intraokulardruck, Brüche in der DescemetMembran, Iris- und Pupillendefekte sowie traumatische Katarakt.

- Bei der FK-Entfernung ist auf eine Schonung der transparenten Strukturen der Kornea in der optischen Achse zu achten.

- Tief liegende inerte Partikel aus Kunststoff oder Glas sollten unter Umständen belassen werden. FK aus organischem Material oder Metall haben ein starkes

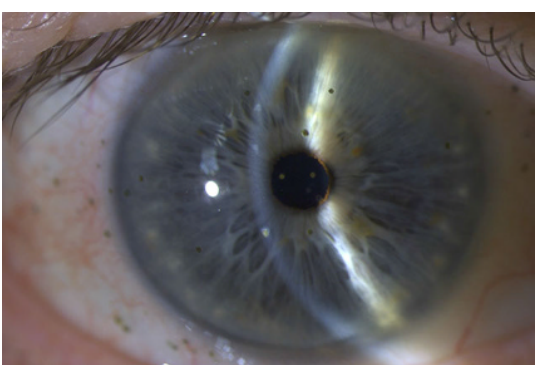

Abb. $5 \Delta$ Der Organbefund nach 4 Monaten mit weiterhin vorhandenem Fremdkörpermaterial, aber in optisch reduzierter Anzahl und Größe

Entzündungspotenzial und müssen entfernt werden.

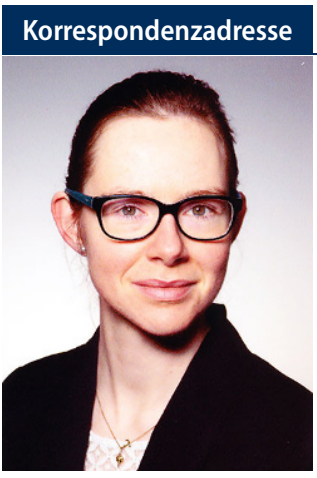

Lisa Lüdtke

Klinik für Augenheilkunde, Universitätsmedizin Greifswald

Greifswald, Deutschland

lisa.luedtke@med.uni-greifswald.de

Funding. Open Access funding enabled and organized by Projekt DEAL.

\section{Einhaltung ethischer Richtlinien}

Interessenkonflikt. A. Stahl erklärt, dass Forschungsunterstützungen mit Novartis und Bayer bestehen sowie den Erhalt von Beratungshonoraren von Novartis, Bayer und Roche, und Vortragshonorare und Reisekostenübernahmen von Novartis, Bayer und Allergan. Er gibt die Teilnahme an Monitoring Boards von Novartis, Bayer und Allergan an sowie den Vorsitz in der Deutschen Retinologischen Gesellschaft und Retina.net.e.V. L. Lüdtke, R. Großjohann, A. Tayar und F. Tost geben an, dass kein Interessenkonflikt besteht.

Für diesen Beitrag wurden von den Autoren keine Studien an Menschen oder Tieren durchgeführt. Für die aufgeführten Studien gelten die jeweils dort angegebenen ethischen Richtlinien. Für Bildmaterial oder anderweitige Angaben innerhalb des Manuskripts, über die Patienten zu identifizieren sind, liegt von innen und/oder ihren gesetzlichen Vertretern eine schriftliche Einwilligung vor.

Open Access. Dieser Artikel wird unter der Creative Commons Namensnennung 4.0 International Lizenz veröffentlicht, welche die Nutzung, Vervielfältigung, Bearbeitung, Verbreitung und Wiedergabe in jeglichem Medium und Format erlaubt, sofern Sie den/die ursprünglichen Autor(en) und die Quelle ordnungsgemäß nennen, einen Link zur Creative Commons Lizenz beifügen und angeben, ob Änderungen vorgenommen wurden.

Die in diesem Artikel enthaltenen Bilder und sonstiges Drittmaterial unterliegen ebenfalls der genannten Creative Commons Lizenz, sofern sich aus der Abbildungslegende nichts anderes ergibt. Sofern das betreffende Material nicht unter der genannten Creative Commons Lizenz steht und die betreffende Handlung nicht nach gesetzlichen Vorschriften erlaubt ist, ist für die oben aufgeführten Weiterverwendungen des Materials die Einwilligung des jeweiligen Rechteinhabers einzuholen.

Weitere Details zur Lizenz entnehmen Sie bitte der Lizenzinformation auf http://creativecommons.org/ licenses/by/4.0/deed.de.

\section{Literatur}

1. http://www.gesetze-im-internet.de/waffg 2002/index.html. Zugegriffen: 19. Juni 2021

2. Camodeca AJ, Anderson EP (2021) Corneal foreign body. StatPearls, Treasure Island (FL) (Available from: https://www.ncbi.nlm.nih.gov/ books/NBK536977)

3. Celebi A, Kilavozuglo A (2016) The role of anterior segment optical coherence tomography in the management of an intra-corneal foreign body. Springer Plus 5(1):1559

4. Frank M, Napp M (2010) Noise trauma induced by a mousetrap-sound pressure level measurement of vole captive bolt devices. J Forensic Sci 3:774-778

5. Gay D, Ritchie J (2013) Ultrasound of penetrating ocular injury in a combat environment. Clin Radiol 68:82-84

6. Guier CP, Stokkermans TJ (2021) Cornea foreign body removal. StatPearls, Treasure Island (FL) (Available from: https://www.ncbi.nlm.nih.gov/ books/NBK554478/)

7. Massoudi D, Malecaze F (2016) Collagens and proteoglycans of the cornea: importance in transparency and visual disorders. Cell Tissue Res 363:337-349

8. Modjtaehedi BS, Rong A (2015) Imaging characteristics of intraocular foreign bodies. Retina 35(1):95-104

9. Parke DW, Flynn HW (2013) Management of intraocular foreign bodies: a clinical fight plan. Can JOphthalmol 48(1):8-12

10. Pauleikhoff L, Reinhard T (2021) Subepitheliale Trübungen im Hornhautstroma nach FKentfernung. Klin Monatsbl Augenheilkd. https://doi.org/ 10.1055/a-1328-2982

11. Shah F, Asif J (2021) Perforating ocular trauma due to shotgun pellet-clinical profile and visual outcome. Indian J Ophthalmol 69(5):1224-1229

12. Smith D, Wrenn K (2002) The epidemiology and diagnosis of penetrating eye injuries. Acad Emerg Med 9(3):209-213 